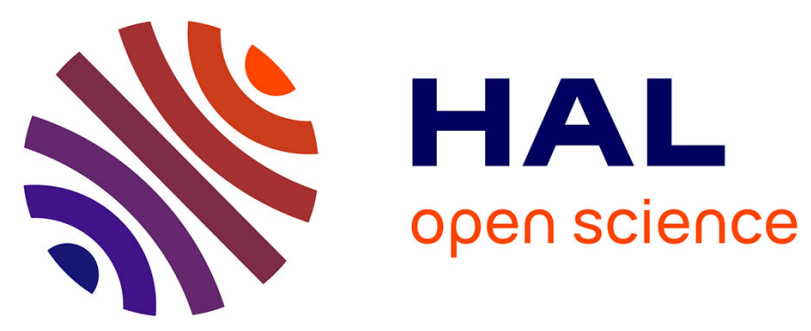

\title{
Nouvelle stratégie de neuroprotection basée sur la chélation conservatrice du fer dans la maladie de Parkinson
}

\author{
Anne-Sophie Rolland, Caroline Moreau, Jean-Christophe Devedjian, \\ Dominique Deplanque, Regis Bordet, N. Carrière, Luc Defebvre, David Devos
}

\section{To cite this version:}

Anne-Sophie Rolland, Caroline Moreau, Jean-Christophe Devedjian, Dominique Deplanque, Regis Bordet, et al.. Nouvelle stratégie de neuroprotection basée sur la chélation conservatrice du fer dans la maladie de Parkinson. Pratique Neurologique - FMC, 2019, Pratique Neurologique - FMC, 10 (2), pp.44-54. 10.1016/j.praneu.2019.01.008 . hal-03262047

\section{HAL Id: hal-03262047 https://hal.univ-lille.fr/hal-03262047}

Submitted on 16 Jun 2021

HAL is a multi-disciplinary open access archive for the deposit and dissemination of scientific research documents, whether they are published or not. The documents may come from teaching and research institutions in France or abroad, or from public or private research centers.
L'archive ouverte pluridisciplinaire HAL, est destinée au dépôt et à la diffusion de documents scientifiques de niveau recherche, publiés ou non, émanant des établissements d'enseignement et de recherche français ou étrangers, des laboratoires publics ou privés. 
Nouvelle stratégie de neuroprotection basée sur la chélation conservatrice du fer dans la maladie de Parkinson

New neuroprotective strategy based on conservative iron chelation in Parkinson's disease

Rolland $\mathrm{AS}^{1}$, Moreau $\mathrm{C}^{2}$, Devedjian $\mathrm{JC}^{3}$, Deplanque $\mathrm{D}^{1}$, Bordet $\mathrm{R}^{1}, \mathrm{~N}_{\text {Carrière }}^{2}$, Defebvre $\mathrm{L}^{2}$, Devos $\mathrm{D}^{1}$ et le FAIRPARK-II study group

Affiliations : ${ }^{1}$ Département de Pharmacologie, CHU Lille, INSERM UMRS_1171, LICEND Université de Lille, France ; ${ }^{2}$ Département de Neurologie, Centre expert de la Maladie de Parkinson, CHU de Lille, INSERM UMRS_1171, LICEND, Université de Lille, France

Adresse de correspondance : Devos David, MD, $\mathrm{PhD}$

Département de Pharmacologie Médicale

Université Lille INSERM 1171

CHU de Lille

F-59037 Lille, France

Email : david.devos@chru-lille.fr 


\section{Résumé}

Il n'existe à l'heure actuelle aucun traitement neuroprotecteur permettant de réduire la progression de la maladie de Parkinson (MP). Au début du vingtième siècle, 3 grandes caractéristiques neuropathologiques ont été décrites : la dégénérescence des neurones dopaminergiques, les corps de Lewy, et l'accumulation en fer au niveau de la substance noire (SN). Cette accumulation en fer ainsi que sa grande toxicité ont été confirmées dans toutes les formes de MP. Par crainte d'induire une anémie, aucune stratégie thérapeutique basée sur la chélation du fer n'avait été développée chez l'humain. Cependant, grâce au concept de chélation conservatrice du fer, nous avons établi une nouvelle stratégie de neuroprotection utilisant la molécule prototype deferiprone et mis en place des essais cliniques actuellement en phase II et III. La découverte récente d'un nouveau mécanisme de mort cellulaire programmée non apoptotique dépendante du fer, appelée ferroptose, qui prédomine dans la MP, permet à la fois d'expliquer le mécanisme d'action de la chélation du fer tout en ouvrant de nouvelles perspectives thérapeutiques de molécules anti-ferroptotiques.

\footnotetext{
Abstract

There is currently no effective neuroprotective therapy to reduce the progression of Parkinson's disease (PD). Early in the twentieth century, it was concomitantly described dopamine depletion, Lewy bodies and iron accumulation in the Substantia Nigra (SN). This accumulation and its toxicity have been since confirmed in all forms of PD. Due to possible risk of anemia, no therapeutic strategy based on iron chelation had been developed in humans. But, thanks to the concept of conservative iron chelation, we have established a new neuroprotection stategy using the prototype molecule deferiprone, and set up clinical trials currently in phase II and III. The recent discovery of a new mechanism of programmed nonapoptotic iron-dependent cell death, called ferroptosis, which predominates in PD, both
} 
explains the mechanism of action of iron chelation and opens up new therapeutic possibilities for anti-ferroptotic drugs.

Mots clés : Maladie de Parkinson, chélation conservatrice du fer, stress oxydant, dopamine, ferroptose, alpha-synucléine

Key words : Parkinson's disease, conservative iron chelation, dopamine oxidative stress, ferroptosis, alpha-synuclein 


\section{LES POINTS ESSENTIELS}

- Une accumulation anormale de fer a été mise en évidence dans la substance noire pars compacta des patients parkinsoniens.

- Une nouvelle forme de mort cellulaire programmée non apoptotique appelée ferroptose pourrait être responsable de certains mécanismes physiopathologiques caractéristiques de la maladie de Parkinson.

- Rétablir l'homéostasie du fer par un système de chélation puis redistribution spécifique semble être un mécanisme prometteur de neuroprotection.

- A l'heure actuelle, la défériprone, est la seule molécule permettant une chélation conservatrice $\mathrm{du}$ fer en cours de validation dans des essais cliniques de phase II. 


\section{INTRODUCTION}

Disposer d'une thérapie pouvant interférer efficacement contre les processus pathologiques conduisant à la maladie de Parkinson (MP) devient primordial pour espérer ralentir sa progression. Un de ces processus est une accumulation anormale de fer labile dans la Substance Noire pars compacta (SNc) [1] qui entraine une production importante de radicaux libres et, à terme, la mort des neurones dopaminergiques. Agir sur cette dyshoméostasie du fer par chélation pourrait constituer une stratégie thérapeutique de neuroprotection. En effet il a été montré dans des modèles animaux que les chélateurs de fer qui éliminaient le fer intracellulaire protégeaient de la mort neuronale. Cependant, ces traitement, conçus pour traiter la sidérose systémique, ne conviennent pas aux patients atteints de la maladie de Parkinson, car ils peuvent entraîner une anémie. Il faut donc se concentrer sur une thérapie chélatrice conservatrice du fer. Nous aborderons successivement dans cette revue, la mise en évidence d'une altération de l'homéostasie du fer dans la maladie de Parkinson ; les potentiels mécanismes cellulaires responsables de cette dyshoméostasie pour ensuite proposer une nouvelle thérapeutique basée sur la recapture et la redistribution ciblée du fer (table 1).

\section{MESURES DU TAUX DE FER DANS LA SNc DE PATIENTS PARKINSONIENS}

L'accumulation de fer a été identifiée sur tissu post-mortem dans la SNc de cerveaux de patients parkinsoniens [1-3] mais également dans des modèles animaux de la maladie de Parkinson [1-5]. Les techniques d'imagerie à hauts champs (IRM) ont permis de confirmer ce taux élevé de fer in vivo, chez le patient parkinsonien, à l'aide d'une séquence quantitative T2* montrant une valeur élevée du R2* (Figure 1) [4,6,7]. Des études longitudinales et des méta-analyses ont mis en évidence une accumulation progressive de fer dans la SNc au cours de la progression de la maladie [6-7]. L'évaluation visuelle de l'hypersignal de la SNc dorsolaterale par séquence SWI (Susceptibility Weighted Imaging) permet de différentier 
patients parkinsoniens et sujets sains et ces observations ont été corroborées par une séquence de mesure dite de relaxation transverse réduite. Plus récemment, une nouvelle approche d'IRM par cartographie de susceptibilité quantitative (QSM) a démontré une sensibilité plus importante des changements de taux de fer non héminiques $[8,9]$ et une nouvelle séquence de contraste de transfert magnétique a identifié une réduction de la densité de neuromélanine spécifiquement dans la SNc [10]. La technique d'ultrason transcraniale qui permet la visualisation de l'hyperéchogénicité de la SNc a démontré une augmentation du taux de fer liée à l'altération des gènes du métabolisme du fer [11].

\section{QUELS PEUVENT ETRE LES MECANISMES CELLULAIRES RESPONSABLES}

\section{DE L'ACCUMULATION DE FER?}

\section{Altération de l'export du fer}

Différentes protéines sont impliquées dans l'exocytose intracellulaire du fer et des données à la fois pré-cliniques et cliniques mettent en lumière leur possible implication dans l'accumulation de fer qui se produit dans la MP :

- la ferroportine, protéine de transport du fer, est diminuée dans les modèles animaux parkinsoniens intoxiqués au 1-methyl-4-phenyl-1,2,3,6-tetrahydropyridine (MPTP) et à la 6-hydroxydopamine (6-OHDA) [1] ;

- $\quad$ une perte de fonction soit de la protéine précurseur amyloide (APP), soit de la de la protéine Tau cause une rétention du fer neuronal aussi bien qu'une perte cellulaire dépendante du fer dans la SNc, et ces deux protéines sont diminuées dans la SNc de patients parkinsoniens. $[3,12]$;

- l'exocytose du fer peut également être assurée par la ceruloplasmine. Les souris déficientes pour cette enzyme développent une augmentation du taux de fer âgedépendant (DFP) ainsi qu'un Parkinson mais ceci peut être réversé par traitement à la 
défériprone (DFP), protéine chélatrice de fer. Une faible activité de la céruloplasmine chez les patients parkinsoniens a été également identifiée dans la $\mathrm{SN}$, le liquide cérébrospinal (LCS) et le sérum plasmatique [1]. Des mutations dans le gène codant pour la céruloplasmine sont également associées à hyperéchogenicité de la SN dans la MP.

\section{Altération du stockage du fer}

La capacité limitée des neurones à séquestrer l'excédent de fer dans les molécules de ferritine [1,2] est compensée par l'expression de neuromélanine, véritable «puits de fer » alternatif [13]. Cependant, de telles capacités pourraient être diminuées dans la MP, conduisant potentiellement à une immunoréactivité augmentée de la ferritine microgliale dans la SN [14]. Des taux élevés de ferritine chargée en fer pourraient, avec le temps, contribuer à une neurodégénérescence liée à l'âge en agissant sur la stabilité du réservoir de fer $[1,2,13]$.

\section{Augmentation de la captation du fer}

Les polymorphismes d'un seul nucléotide du gène de la transferrine (Tf) et de son récepteur (TfR), identifiés dans des études de cas contrôle de la MP, pourraient avoir un rôle protecteur via des modifications du transport de fer lié à la Tf dans la cellule [15]. La lactoferrine et son récepteur peuvent également jouer un rôle similaire [16]. Enfin, l'accumulation de fer dans la SNc de patients et de souris intoxiquées au MPTP est corrélée avec une augmentation de l'import du fer, le transport de métal divalent de type 1 (DMT1) [17].

\section{Y'A T'IL UNE INTERACTION FER-ALPHA-SYNUCLEINE?}

Le fer induit l'agrégation importante d'alpha-synucléine ( $\alpha$-synucléine) sous forme d'inclusions intracellulaires appelées corps de Lewy [18]. En accord avec l'accumulation de fer, les mesures de Quantitative Susceptibility Mapping (QSM) suivent le pattern de distribution de l'alpha-synucléine dans la MP [8]. Il a été de plus démontré que le fer pouvait 
augmenter la traduction donc le niveau de protéine via sa région promotrice [18]. Plus récemment il a été suggéré un rôle de l' $\alpha$-synucléine dans la modulation de l'homéostasie du fer. En effet, une réduction du taux d' $\alpha$-synucléine altèrerait les capacités du récepteur à la transferrine (TfR) à importer le fer dans la cellule et indiquerait que l'alpha-synucléine peut moduler l'endocytose médiée par les clatherines [18]. Une alimentation néonatale riche en fer dans un modèle de souris transgénique surexprimant l' $\alpha$-synucléine humaine porteuse de la mutation A53T exacerbe à la fois les phénotypes moteurs et non moteurs de la MP. En conséquence, la chélation du fer réduit la quantité d'agrégats insolubles d' $\alpha$-synucléine et sauve les déficits comportementaux [19] dans des modèles murins génétiques de la MP.

\section{LA FERROPTOSE- UNE NOUVELLE FORME DE MORT CELLULAIRE DEPENDANTE DU FER- POURRAIT APPORTER DE NOUVELLES PERSPECTIVES THERAPEUTIQUES DANS LA MP}

Une nouvelle forme de mort cellulaire, la ferroptose, récemment mise en évidence, a de fortes implications dans la neuropathologie de la MP. Elle apparait être sélectivement provoquée par un mécanisme dépendant du fer avec comme conséquence une peroxydation lipidique, une diminution spécifique de la glutathione peroxidase-4 altérant la protection de la glutathione, une mitochondriopathie ainsi que des modifications morphologiques distinctes différentes de celles des autres formes de mort cellulaires (i.e. apoptose, nécrose et autophagie) [20]. L'inhibition du système xCT cystéine/glutamate pendant la ferroptose empêche par conséquence l'import de cystine dans la cellule et conduit à une diminution de la synthèse de glutathione et augmente la disponibilité cellulaire de fer labile qui catalyse la peroxydation lipidique (Figure 2) [20]. La ferroptose est associée à des changements pathogéniques observés dans la MP, aussi bien que dans les modèles pro-oxydants classiques in vitro et in vivo [21]. Cela inclut une augmentation du taux de fer dans la $\mathrm{SN}$, une mitochondropathie, 
une diminution du taux de glutathione, une peroxydation lipidique, une production de radicaux libres et une oxydation de la dopamine [21]. La ferroptose peut être reversée indirectement par la chélation du fer (i.e avec de la deferiprone-DFP-) [20,21], corroborant ainsi l'implication du fer dans l'initiation de ce type de mort cellulaire. De manière importante, un panel d'inhibiteurs directs la ferroptose (e.g. liproxstatin-1) a été récemment désigné avec des implications thérapeutiques prometteuses.

\section{UNE NOUVELLE STRATEGIE THERAPEUTIQUE DE CHELATION CONSERVATRICE DU FER BASEE SUR LA RECAPTURE ET LA REDISTRIBUTION DU FER}

L'implication de la sidérose et de la toxicité du fer a largement été basée sur les effets protecteurs de la chélation du fer dans les modèles cellulaires et animaux $[1,2,6,7,22,23]$. Cependant, pour que ces chélateurs soient cliniquement efficaces dans l'accumulation de fer ou sidérose, ils doivent avoir une accessibilité spécifique aux compartiments ou cellules concernées afin d'éviter que des zones non surchargées en fer voir déficitaires soient victime de la chélation [24]. Différentes molécules avec des caractéristiques chélatrices de fer (i.e. deferoxamine, clioquinol, VK28, M30 et flavonoid polyphenol derive de lantes) ont été étudiées mais n'ont pas été portées en essai clinique.

La DFP est actuellement la seule molécule qui présente les propriétés de chélation conservatrice du fer de par ses capacités à traverser les membranes et en particulier la barrière hématoencéphalique, mais aussi de part ses capacités à chélater les composants du pool de fer labile cellulaire dans le tissu cérébral [24]. La DFP a cette capacité remarquable de réduire l'excès de fer intracellulaire et intra-mitochondriale puis de pouvoir le redonner en extracellulaire à la transferrine (le transporteur naturelle du fer) permettant ainsi de 
redistribuer le fer dans l'ensemble de l'organisme pour éviter des déficit focaux ou généraux (anémie) (Figure 3) [24].

La stratégie de redistribution conservative a été appliquée dans la MP par traitement à la DFP a de faible dose orale $(30 \mathrm{mg} / \mathrm{kg} / \mathrm{j})$ [4]. Une étude pilote avec des patients parkinsoniens à un stade débutant de la maladie selon un paradigme de début retardé (prétraitement pendant 6 mois de DFP ou de placebo suivi par 12 mois de DFP pour tous) a montré une réduction significative du taux de fer dans la SNc, plus particulièrement dans le groupe qui a commencé la DFP précocement. Comparé au groupe placebo, cet effet perdure pendant tout le traitement (18 mois). Un effet bénéfique clinique concomitant a été noté à 6 mois avec une amélioration de 3 points de l'échelle MDS-UPDRS pour le groupe traité précocement $(21,6 \pm 8)$ comparé au groupe traité tardivement $(24 \pm 6)$. Egalement, à 12 mois, ces patients "précoces" avaient un handicap moteur plus faible (1 point de l'UPDRS III : 21,3 \pm 8 ) comparé aux patients "tardifs" $(22,8 \pm 6)$, suggérant un effet « disease modifier » sur la progression de la maladie [4]. L'effet chélateur conservateur du fer se reflète par l'absence de perte de fer systémique chez les patients montrant un taux de fer normal qui n'était pas altéré après 18 ou 24 mois de traitement par la DFP (hormis une légère réduction de ferritine dans le sang et le LCS). Des résultats cliniques encourageants ont été récemment confirmés dans un autre essai placebo randomisé en double aveugle. Dans cet essai clinique de petit taille, la DFP réduit le contenu de fer des noyaux dentelé et caudé et indique une tendance à l'amélioration du score moteur MDS-UPDRS et de la qualité de vie [25]. Dans les deux essais cliniques, la DFP a un bon profil de sécurité malgré la nécessité d'avoir un bilan sanguin chaque semaine afin de monitorer une neutropénie réversible qui arrive chez 1 à $3 \%$ de patients traités avec la DFP.

Ces résultats prometteurs ont désormais conduit à un essai clinique randomisé européen, multicentrique de phase II, placebo, dont le but est d'évaluer si la DFP peut ralentir la progression de la maladie chez les patients parkinsoniens (www.fairpark2.eu). 388 patients 
parkinsoniens de novo vont être randomisés pour recevoir soit la DFP (30 mg/kg/j, 2 doses par jour) soit le placebo et ce pour une durée de 9 mois. Tous ces patients vont ensuite être monitorés pendant 1 mois. Pour évaluer le potentiel effet modificateur de maladie de la DFP, le premier critère d'efficacité va être le score total moteur MDS-UPDRS, les symptômes moteurs et non moteurs ainsi que leur activité au quotidien. Les critères secondaires vont être la cognition, la qualité de vie, l'activité motrice continue quantitative à la maison et des questionnaires économiques de santé. De potentiels biomarqueurs prédictifs et théranostiques d'efficacité et de sécurité vont également être analyses (i.e. marqueurs de l'homéostasie du fer, de la dopamine et d'alpha-synucléine). Ceci implique également des mesures en imagerie du contenu en fer (IRM R2*/QSM et ultrason transcranienne) et du contenu en dopamine (imagerie SPECT du transporteur de la dopamine). Depuis l'initiation de l'essai clinique FAIR-PARK-II, deux essais de phase II sont conduits en Europe, en Australie et au Canada pour déterminer la meilleure dose de DFP, de 10 à $40 \mathrm{mg} / \mathrm{kg} / \mathrm{jour}$ et évaluer l'effet clinique sur la même période.

\section{CONCLUSIONS ET FUTURES DIRECTIONS}

La dyshoméostasie du fer cellulaire a été impliquée dans une grande variété de maladies neurodégénératives autre que la MP, telle que la maladie d'Alzheimer (MA; élévation en fer corticale), la sclérose latérale amyotrophique (SLA; taux de fer élevé dans les voies du motoneurones) et les rares maladies d'accumulation de fer dans le cerveau (Neurodegeneration with Brain Iron Accumulation). La distribution altérée du fer pourrait représenter une forme pathologique de sidérose régionale susceptible d'être traitée par un chélateur conservateur du fer basé sur une redistribution du fer médiée par une drogue, actuellement en évaluation clinique pour la MP, la neurodégénération associée à la pantothénate kinase de type 2, SLA et MA. Le principe est de ralentir la progression de la 
maladie, mais des stratégies complémentaires pourraient également bloquer les voies de mort cellulaire dépendantes du fer telle que la ferroptose ou stimuler les voies de signalisation de réparation cellulaire via la formation de glutathione ou l'inhibition de proly-hydroxylases dépendante du fer [4,21]. L'espoir actuel est qu'avec le développement de nouveaux biomarqueurs en imagerie avec des séquences sensibles au fer, les essais cliniques tels que ceux présentés ici de chélation conservatrice du fer pourraient changer la prise en charge de la MP.

\section{REMERCIEMENTS}

Les auteurs remercient le soutien du CHU de Lille, le réseau de recherche Clinique NSPark/FCRIN, la Commission Européenne pour le financement $\mathrm{N}^{\circ} 633190$ du programme H2020; NCT02655315. Les auteurs remercient également la Fédération de la Recherche Clinique du CHU de Lille, Pauline Guyon, Marcellin Bellonet, Edouard Millois, Stéphanie Le Naour, Amelie Michon, Aurélie Rabier et ApoPharma qui fournit gratuitement les traitements de l'étude.

\section{DECLARATION DE LIENS D'INTERET}

David Devos a mené deux études pilotes académiques avec la deferiprone fournit gratuitement par Apopharma (FAIRPARK-I and SAFE-FAIR-ALS-I). Il mène deux larges étude académiques avec la deferiprone fournit gratuitement par Apopharma (FAIRPARK-II and FAIR-ALS-II). Apopharma effectue son propre développement clinique de la deferiprone avec deux études de phase II en cours et une phase III programmée dans la maladie de Parkinson. 
Anne Sophie Rolland, Caroline Moreau, Jean-Christophe Devedjian, Dominique Deplanque,

Régis Bordet, Nicolas Carrière, Luc Defebvre n'ont pas de conflit d'intérêt en relation avec cet article.

FAIRPARK II study group (with the support of the Lille University Hospital and NSPark/FCRIN clinical research network, www.fairpark2.eu; funded by European commission grant $N^{\circ} 633190$ of the H2020 program; NCT02655315)

- Abbruzzese Giovanni University of Genove Italy

- Allain Marie-Anne ALLAIN CHU Lille France

- Anheim Mathieu, Department of Movement Disorders and Neurology, NS-Park/FCRIN Network, CHU Strasbourg, Strasbourg, France

- Bakker Martijn University Nijmegen Medical Center, Donders Institute Brain Cognition \& Behaviour Center for Neurosciences The Netherland

- Balzer-Geldsetzer Monika ipps University Hospital Essen, GermanyUniversitat Marburg

- Bargalló Núria Magnetic Resonance Unit, Neurorradiology Section, Centre de Diagnòstic per la Imatge (CDI), IDIBAPS, Hospital Clínic, Barcelona, Catalonia, Spain

- Barone Paolo University of Salerno Italy

- Basenau Sandra, Philipps Universitat Marburg, Germany

- Benchetrit Eve ICM, Hôpital Pitié-Salpêtrière, Paris, France

- Berg Daniela, Department of Neurology, Christian-Albrechts-University of Kiel, Kiel, Germany

- Bloem Bas University Nijmegen Medical Center, Donders Institute Brain Cognition \& Behaviour Center for Neurosciences The Netherland

- Boraud Thomas Université de Bordeaux, Institut des Maladies Neurodégénératives, UMR CNRS 5293 and Department of Neurology, NS-Park/FCRIN Network, CHU de Bordeaux, Bordeaux, France

- Bordet Regis, University de Lille, CHU de Lille, INSERM UMRS_1171, Service de Pharmacologie Clinique LICEND COEN Center Lille, France

- Bouca Raquel Instituto de Medicina Molecular Lisboa Portugal

- Bourdain Frédéric, Hôpital Foch, Suresnes, France

- Bouzas Jimena European Clinical Research Infrastructure Network-ERIC, France

- Brefel-Courbon Christine, CHU de Toulouse, INSERM; Centre d'Investigation Clinique CIC1436, Services de Neurologie et de Pharmacologie Clinique, NS-Park/FCRIN Network, Toulouse, France

- Bubenheim Michael CHU-Hôpitaux de Rouen France

- Burn David, Faculty of Medical Sciences Newcastle University United Kingdom

- Bush Ashley I., Oxidation Biology Unit, The Florey Institute of Neuroscience and Mental Health, The University of Melbourne, Parkville, Victoria, Australia

- Cabantchik Ioav, Della Pergola Chair, Alexander Silberman Institute of Life Sciences, Hebrew University, Jerusalem, 91904, Israel

- Calvas Fabienne, CHU de Toulouse, INSERM; Centre d'Investigation Clinique CIC1436, Services de Neurologie et de Pharmacologie Clinique, NS-Park/FCRIN Network, Toulouse, France 
- Cámara Ana Parkinson's disease \& Movement Disorders Unit, Neurology Service, Hospital Clínic de Barcelona, IDIBAPS, University of Barcelona, CIBERNED, Barcelona, Catalonia, Spain

- Carrière Nicolas, University de Lille, CHU de Lille, INSERM UMRS 1171, Service de Neurologie NS-Park/FCRIN Network LICEND COEN Center Lille, France

- Chaigneau Véronique, NS-Park/FCRIN Network, Toulouse, France

- Compta Yaroslau, Parkinson's disease \& Movement Disorders Unit, Neurology Service, Hospital Clínic de Barcelona, IDIBAPS Barcelona, Catalonia, Spain

- Connelly John, ApoPharma Inc., Toronto, Canada

- Cormier-Dequaire Florence Hôpital Pitié-Salpêtrière, Paris, France

- Corvol Jean-Christophe, Sorbonne Universités, UPMC Univ Paris 06, and INSERM UMRS_1127 and CIC_1422, and CNRS UMR_7225, and AP-HP, and ICM, Hôpital PitiéSalpêtrière, NS-Park/FCRIN Network, Département des maladies du système nerveux, Paris, France

- Cranston Amy University of Newcastle upon Tyne UK

- De Bie Rob, Academisch Medisch Centrum Universiteit van Amsterdam.Amsterdam, Netherlands

- De Marzi Roberto, Department of Neurology, Medical University Innsbruck, Innsbruck, Austria

- Defebvre Luc, University de Lille, CHU de Lille, INSERM UMRS_1171, Service de Neurologie NS-Park/FCRIN Network LICEND COEN Center Lille, France

- Degos Bertrand Hôpital Pitié-Salpêtrière, Paris, France

- Demotes Jacques, European Clinical Research Infrastructure Network-ERIC, France

- Dellapina Estelle, NS-Park/FCRIN Network, Toulouse, France

- Deplanque Dominique, University de Lille, CHU de Lille, INSERM UMRS 1171, Service de Pharmacologie Clinique et CIC-CHU Lille, LICEND COEN Center Lille, France

- Devedjian Jean-Christophe, University de Lille, CHU de Lille, INSERM UMRS_1171, NSPark/FCRIN Network LICEND COEN Center Lille, France

- Devos David, University de Lille, CHU de Lille, INSERM UMRS_1171, Service de Pharmacologie Clinique et service de Neurologie NS-Park/FCRIN Network LICEND COEN Center Lille, France

- Dexter David, Imperial College London London United Kingdom

- Dodel Richard, University Hospital Essen, GermanyPhilipps Universitat Marburg Germany

- Dongmo Carole Hôpital Pitié-Salpêtrière, Paris, France

- Duce James, School of Biomedical Sciences, Faculty of Biological Sciences, University of Leeds, Leeds, West Yorkshire, UK \& Oxidation Biology Unit, The Florey Institute of Neuroscience and Mental Health, The University of Melbourne, Parkville, Victoria, Australia

- Duhamel Alain, CHU Lille France

- Dupouy Julia, CHU de Toulouse, INSERM; Centre d'Investigation Clinique CIC1436, Services de Neurologie et de Pharmacologie Clinique, NS-Park/FCRIN Network, Toulouse, France

- Durif Franck, Department of Movement Disorders and Neurology, NS-Park/FCRIN Network, CHU Clermont-Ferrand, Clermont-Ferrand, France

- Dusek Petr, Dept. of Neurology, Charles University, Prague, Czech Republic

- Eusebio Alexandre, Department of Neurology and Movement Disorders - APHM Timone University Hospital and Institut de Neurosciences de la Timone, NS-Park/FCRIN Network, AMU-CNRS UMR 7289, Marseille, France

- Eyvrard Frédéric, Pharmacy, CHU de Toulouse, Toulouse, France 
- Fernández Manel Parkinson's disease \& Movement Disorders Unit, Neurology Service, Hospital Clínic de Barcelona, IDIBAPS, University of Barcelona, CIBERNED, Barcelona, Catalonia, Spain

- Ferreira Joaquim, Instituto de Medicina Molecular Lisboa Portugal

- Forni Gian Luca, Ospedia Galliera Italy

- Foubert-Samier Alexandra Université de Bordeaux, Institut des Maladies

Neurodégénératives, UMR CNRS 5293 and Department of Neurology, NS-Park/FCRIN

Network, CHU de Bordeaux, Bordeaux, France

- Fradette Caroline, ApoPharma Inc., Toronto, Canada

- Fréville Laëtitia, Centre pour l'Acquisition et le Traitement des Images (www.catineuroimaging.com); Sorbonne Universités, UPMC Univ Paris 06, CNRS, INSERM, Laboratoire d'Imagerie Biomédicale, F-75013, Paris, France

- Galitzky Monique, CHU de Toulouse, INSERM; Centre d'Investigation Clinique CIC1436, Services de Neurologie et de Pharmacologie Clinique, NS-Park/FCRIN Network, Toulouse, France

- Gaudebout Cecile Hôpital Pitié-Salpêtrière, Paris, France

- Gelé Patrick CHU de Lille CHU Lille France

- Giladi Nir Tel Aviv Sourasky Medical Center Israel

- Grabli David Hôpital Pitié-Salpêtrière, Paris, France

- Grolez Guillaume University de Lille, CHU de Lille, INSERM UMRS_1171, Service de Neurologie NS-Park/FCRIN Network LICEND COEN Center Lille, France

- Guyon Pauline CHU Lille France

- Habert Marie-Odile, Sorbonne Universités, UPMC Univ Paris 06, CNRS, INSERM, Laboratoire d'Imagerie Biomédicale, AP-HP, Hôpital Pitié-Salpêtrière, Département de Médecine Nucléaire, F-75013, Paris, France

- Harroch Estelle, CHU de Toulouse, INSERM; Centre d'Investigation Clinique CIC1436, Services de Neurologie et de Pharmacologie Clinique, Toulouse, France

- Hartmann Andreas Hôpital Pitié-Salpêtrière, Paris, France

- Hirsch Denise, INSERM - Transfert SA Paris

- Hopfner Franziska Department of Neurology, Christian-Albrechts-University of Kiel, Kiel, Germany

- Jurado Camille, Pharmacy, CHU de Toulouse, Toulouse, France

- Kaiser Andreas Medizinische Universitat Innsbruck Austria

- Klaus Seppi Medizinische Universitat Innsbruck

- Kouassi Nadège, CHU de Toulouse, INSERM; Centre d'Investigation Clinique CIC1436, Services de Neurologie et de Pharmacologie Clinique, Toulouse, France

- Labreuch Julien CHU de Lille CHU Lille France

- Lacomblez Lucette Hôpital Pitié-Salpêtrière, Paris, France

- Lanthaler Barbara Medizinische Universitat Innsbruck Austria

- Le Forestier Nadine Assistance Publique - Hôpitaux de Paris France

- Le Naour Stéphanie INSERM - Transfert SA Paris France

- Le Toullec Benjamin Hôpital Pitié-Salpêtrière, Paris, France

- Locatelli Maxime, Centre pour l'Acquisition et le Traitement des Images (www.catineuroimaging.com); ICM Institut du Cerveau et de la Moelle épinière, CNRS UMR7225, INSERM U1127, UPMC, F-75013, Paris, France

- Lomeña Francisco Nuclear Medicine Department, Hospital Clínnic, IDIBAPS, University of Barcelona, CIBERSAM, Barcelona, Catalonia, Spain

- Longato Nadine Department of Movement Disorders and Neurology, NS-Park/FCRIN Network, CHU Strasbourg, Strasbourg, France 
- Lützen Ulf Department of Nuclear Medicine, Molecular Diagnostic Imaging and Therapy, University Hospital of Schleswig-Holstein (UKSH), Campus Kiel, Germany

- Maetzler Corina Department of Neurology, Christian-Albrechts-University of Kiel, Kiel, Germany

- Maetzler Walter, Department of Neurology, Christian-Albrechts-University of Kiel, Kiel, Germany

- Mahlknecht Philipp Medizinische Universitat Innsbruck Austria

- Mangone Graziella Hôpital Pitié-Salpêtrière, Paris, France

- Mariani Louise-Laure Hôpital Pitié-Salpêtrière, Paris, France

- Marques Ana, Department of Movement Disorders and Neurology, NS-Park/FCRIN Network, CHU Clermont-Ferrand, Clermont-Ferrand, France

- Matthias Löhle, MD, Department of Neurology, University of Rostock, Rostock, Germany

- Meissner Wassilios G., Université de Bordeaux, Institut des Maladies Neurodégénératives, UMR CNRS 5293 and Department of Neurology, NS-Park/FCRIN Network, CHU de Bordeaux, Bordeaux, France

- Michon Amelie, European Clinical Research Infrastructure Network-ERIC, France

- Moreau Caroline, University de Lille, CHU de Lille, INSERM UMRS_1171, Service de Neurologie NS-Park/FCRIN Network LICEND COEN Center Lille, France

- Nardocci Nardo Nardocci Istitu Besta Italy

- Nosal Florence CHU de Lille France

- Nyholm Dag Uppsala University Sweden

- Oeckl Patrick Universitaet Ulm Germany

- Ory Fabienne, CHU de Toulouse, INSERM; Centre d'Investigation Clinique CIC1436, Services de Neurologie et de Pharmacologie Clinique, Toulouse, France

- Otto Markus, Universitaet Ulm Germany

- Ouk Thavarak University de Lille, CHU de Lille, INSERM UMRS_1171, Service de Neurologie NS-Park/FCRIN Network LICEND COEN Center Lille, France

- Pavese Nicola University of Newcastle upon Tyne UK

- Peball Marina Medizinische Universitat Innsbruck Austria

- Phillips Clélie Department of Movement Disorders and Neurology, NS-Park/FCRIN Network, CHU Strasbourg, Strasbourg, France

- Pineau Fanny Hôpital Pitié-Salpêtrière, Paris, France

- Planellas Lluís Parkinson's disease \& Movement Disorders Unit, Neurology Service, Hospital Clínic de Barcelona, IDIBAPS, University of Barcelona, CIBERNED, Barcelona, Catalonia, Spain

- Poewe Werner, Department of Neurology, Medical University Innsbruck, Innsbruck, Austria

- Pop-Ilieva Lydia, ApoPharma Inc., Toronto, Canada

- Post Bart, Radboud University Nijmegen Medical Center, Donders Institute Brain Cognition $\&$ Behaviour Center for Neurosciences The Netherland

- Rabier Aurélie Inserm NS-Park/FCRIN Network, Toulouse, France

- Rascol Olivier, Université de Toulouse, UPS, CHU de Toulouse, INSERM; Centre d'Investigation Clinique CIC1436, Services de Neurologie et de Pharmacologie Clinique, UMR TONIC, NS-Park/FCRIN Network, NeuroToul COEN Center, Toulouse, France

- Riedel Christian Department of Neuroradiology, Christian Albrechts University, Kiel, Germany

- Rodrigues Maura Hôpital Pitié-Salpêtrière, Paris, France

- Rose Christian Hopital Saint-Vincent-de-Paul Lille France

- Roullet-Solignac Isabelle Movement Disorders Unit, Hopital Neurologique, Hospices Civils de Lyon, Université de Lyon, Université Claude Bernard Lyon 1, CNRS Institut des 
Sciences Cognitives, Centre de Neurosciences, Cognitives, UMR 5229, NS-Park/FCRIN Network, Bron Lyon France

- Rozova Anna, ApoPharma Inc., Toronto, Canada

- Růžička Evžen, Dept. of Neurology, Charles University, Prague, Czech Republic

- Salis Alexandra, Inserm NS-Park/FCRIN Network, Toulouse, France

- Schäffer Eva Department of Neurology, University Hospital Schleswig-Holstein, ChristianAlbrechts University Kiel, Germany

- Scherfler Christoph Department of Neurology, Medical University Innsbruck, Innsbruck, Austria

- Schiefermeier Natalia, Department of Neurology, Medical University Innsbruck, Innsbruck, AustriaSeppi Klaus Department of Neurology, Medical University Innsbruck, Innsbruck, AustriaSmagghe Delphine, INSERM - Transfert SA Paris France

- Silva Tânia, Instituto de Medicina Molecular Lisboa Portugal

- Socha Julie Hôpital Pitié-Salpêtrière, Paris, France

- Souyris Corinne, CHU de Toulouse, INSERM; Centre d'Investigation Clinique CIC1436, Services de Neurologie et de Pharmacologie Clinique, Toulouse, France

- Spampinato Umberto Université de Bordeaux, Institut des Maladies Neurodégénératives, UMR CNRS 5293 and Department of Neurology, NS-Park/FCRIN Network, CHU de Bordeaux, Bordeaux, France

- Spino Michael, ApoPharma Inc., Toronto, Canada

- Steel Alison University of Newcastle upon Tyne UK

- Sweta Bajaj Medizinische Universitat Innsbruck Austria

- Thalamas Claire, CHU de Toulouse, INSERM; Centre d'Investigation Clinique CIC1436, Services de Neurologie et de Pharmacologie Clinique, Toulouse, France

- Teodor Danaila Movement Disorders Unit, Hopital Neurologique, Hospices Civils de Lyon, Université de Lyon, Université Claude Bernard Lyon 1, CNRS Institut des Sciences Cognitives, Centre de Neurosciences, Cognitives, UMR 5229, NS-Park/FCRIN Network, Bron Lyon France

- Teresa Anna Instituto de Medicina Molecular Lisboa Portugal

- Thobois Stéphane, Movement Disorders Unit, Hopital Neurologique, Hospices Civils de Lyon, Université de Lyon, Université Claude Bernard Lyon 1, CNRS Institut des Sciences Cognitives, Centre de Neurosciences, Cognitives, UMR 5229, NS-Park/FCRIN Network, Bron Lyon FranceFrance

- Tison François Université de Bordeaux, Institut des Maladies Neurodégénératives, UMR CNRS 5293 and Department of Neurology, NS-Park/FCRIN Network, CHU de Bordeaux, Bordeaux, France

- Tolosa Eduardo, Parkinson's disease \& Movement Disorders Unit, Neurology Service, Hospital Clínic de Barcelona, IDIBAPS, University of Barcelona, CIBERNED, Barcelona, Catalonia, Spain

- Tranchant Christine, Department of Movement Disorders and Neurology, NS-Park/FCRIN Network, CHU Strasbourg, Strasbourg, France

- Tricta Fernando, ApoPharma Inc., Toronto, Canada

- Trifirò Gianluca Trifirò University of Messina \& Erasmus Medical Center, Rotterdam The Netherlands

- Uwe Walter, MD, Department of Neurology, University of Rostock, Rostock, Germany

- Vidailhet Marie Hôpital Pitié-Salpêtrière, Paris, France

- Wang Yi, PhD, Departments of Radiology and Biomedical Engineering, Cornell University, New York, USA

- Werkmann Mario Medizinische Universitat Innsbruck Austria 
- Yilmas Rezzak Department of Neurology, University Hospital Schleswig-Holstein, Christian-Albrechts University Kiel, Germany

- You Hana Hôpital Pitié-Salpêtrière, Paris, France

- Zeuner Kirsten Department of Neurology, University Hospital Schleswig-Holstein, Christian-Albrechts University Kiel, Germany

- The investigators of the 24 centres 


\section{REFERENCES}

[1] Ward RJ, Zucca FA, Duyn JH, Crichton RR, Zecca L. The role of iron in brain ageing and neurodegenerative disorders. The Lancet Neurology 2014;13:1045-60

[2] Belaidi AA, Bush AI. Iron neurochemistry in Alzheimer's disease and Parkinson's disease: targets for therapeutics. Journal of Neurochemistry 2016;139:179-97

[3] Ayton S, Lei P, Duce JA, Wong BXW, Sedjahtera A, Adlard PA, et al. Ceruloplasmin dysfunction and therapeutic potential for Parkinson disease: Ceruloplasmin in PD. Annals of Neurology 2013;73:554-9

[4] Devos D, Moreau C, Devedjian JC, Kluza J, Petrault M, Laloux C, et al. Targeting Chelatable Iron as a Therapeutic Modality in Parkinson's Disease. Antioxidants \& Redox Signaling 2014;21:195-210.

[5] Kaur D, Yantiri F, Rajagopalan S, Kumar J, Mo JQ, Boonplueang R, et al. Genetic or Pharmacological Iron Chelation Prevents MPTP-Induced Neurotoxicity In Vivo: A Novel Therapy for Parkinson's Disease n.d.:11.

[6] Ulla M, Bonny JM, Ouchchane L, Rieu I, Claise B, Durif F. Is R2* a New MRI Biomarker for the Progression of Parkinson's Disease? A Longitudinal Follow-Up. PLoS ONE 2013;8:e57904

[7] Hopes L, Grolez G, Moreau C, Lopes R, Ryckewaert G, Carrière N, et al. Magnetic Resonance Imaging Features of the Nigrostriatal System: Biomarkers of Parkinson's Disease Stages? PLOS ONE 2016;11:e0147947

[8] Acosta-Cabronero J, Cardenas-Blanco A, Betts MJ, Butryn M, Valdes-Herrera JP, Galazky I, et al. The whole-brain pattern of magnetic susceptibility perturbations in Parkinson's disease. Brain 2017;140:118-31

[9] Wang Y, Spincemaille P, Liu Z, Dimov A, Deh K, Li J, et al. Clinical quantitative susceptibility mapping (QSM): Biometal imaging and its emerging roles in patient care: Clinical QSM Biometals. Journal of Magnetic Resonance Imaging 2017;46:951-71

[10] Huddleston DE, Langley J, Sedlacik J, Boelmans K, Factor SA, Hu XP. In vivo detection of lateral-ventral tier nigral degeneration in Parkinson's disease: MRI of Lateral-Ventral SNc in PD. Human Brain Mapping 2017;38:2627-34

[11] Zecca L, Berg D, Arzberger T, Ruprecht P, Rausch WD, Musicco M, et al. In vivo detection of iron and neuromelanin by transcranial sonography: A new approach for early detection of substantia nigra damage. Movement Disorders 2005;20:1278-85

[12] Lei P, Ayton S, Finkelstein DI, Spoerri L, Ciccotosto GD, Wright DK, et al. Tau deficiency induces parkinsonism with dementia by impairing APP-mediated iron export. Nature Medicine 2012;18:291-5

[13] Hare DJ, Double KL. Iron and dopamine: a toxic couple. Brain 2016;139:1026-35

[14] Wu K-C, Liou H-H, Kao Y-H, Lee C-Y, Lin C-J. The critical role of Nramp1 in degrading $\alpha$-synuclein oligomers in microglia under iron overload condition. Neurobiology of Disease 2017;104:61-72

[15] Rhodes SL, Buchanan DD, Ahmed I, Taylor KD, Loriot M-A, Sinsheimer JS, et al. Pooled analysis of iron-related genes in Parkinson's disease: Association with transferrin. Neurobiology of Disease 2014;62:172-8

[16] Faucheux BA, Nillesse N, Damier P, Spik G, Mouatt-Prigent A, Pierce A, et al. Expression of lactoferrin receptors is increased in the mesencephalon of patients with Parkinson disease. Proceedings of the National Academy of Sciences 1995;92:9603-7

[17] Salazar J, Mena N, Hunot S, Prigent A, Alvarez-Fischer D, Arredondo M, et al. Divalent metal transporter 1 (DMT1) contributes to neurodegeneration in animal models of Parkinson's disease. Proceedings of the National Academy of Sciences 2008;105:18578-83 
[18] Duce JA, Wong BX, Durham H, Devedjian J-C, Smith DP, Devos D. Post translational changes to $\alpha$-synuclein control iron and dopamine trafficking; a concept for neuron vulnerability in Parkinson's disease. Molecular Neurodegeneration 2017;12

[19] Carboni E, Tatenhorst L, Tönges L, Barski E, Dambeck V, Bähr M, et al. Deferiprone Rescues Behavioral Deficits Induced by Mild Iron Exposure in a Mouse Model of Alpha-Synuclein Aggregation. NeuroMolecular Medicine 2017;19:309-21

[20] Dixon SJ, Lemberg KM, Lamprecht MR, Skouta R, Zaitsev EM, Gleason CE, et al. Ferroptosis: An Iron-Dependent Form of Nonapoptotic Cell Death. Cell 2012;149:106072

[21] Do Van B, Gouel F, Jonneaux A, Timmerman K, Gelé P, Pétrault M, et al. Ferroptosis, a newly characterized form of cell death in Parkinson's disease that is regulated by PKC. Neurobiology of Disease 2016;94:169-78

[22] Weinreb O, Mandel S, Youdim MBH, Amit T. Targeting dysregulation of brain iron homeostasis in Parkinson's disease by iron chelators. Free Radical Biology and Medicine 2013;62:52-64

[23] Workman DG, Tsatsanis A, Lewis FW, Boyle JP, Mousadoust M, Hettiarachchi NT, et al. Protection from neurodegeneration in the 6-hydroxydopamine (6-OHDA) model of Parkinson's with novel 1-hydroxypyridin-2-one metal chelators. Metallomics 2015;7:867-76

[24] Cabantchik ZI, Munnich A, Youdim MB, Devos D. Regional siderosis: a new challenge for iron chelation therapy. Frontiers in Pharmacology 2013;4

[25] Martin-Bastida A, Ward RJ, Newbould R, Piccini P, Sharp D, Kabba C, et al. Brain iron chelation by deferiprone in a phase 2 randomised double-blinded placebo controlled clinical trial in Parkinson's disease. Scientific Reports 2017;7. 


\section{TABLE I : principaux points d'intérêts pour les neurologistes}

6-OHDA : 6-hydroxydopamine; IRM : imagerie par résonnance magnétique ; MP = maladie de Parkinson; MA : maladie d'Alzheimer; $\mathrm{SN} c$ : Substance Noire pars compacta; $\mathrm{SN} r$ : Substance Noire pars reticulata; PKAN-2: Pantothenate Kinase-Associate Neurodegeneration type 2; QSM: Quantitative Susceptibility Mapping; VTA: Aire Tegmentale Ventrale; SWI: Susceptibility weighted imaging; SLA: sclérose laterale amyotrophique 
FIGURE 1 : Accumulation de fer dans la SN de patients parkinsoniens

L'analyse post-mortem de mésencéphale de sujets sains et patients parkinsoniens montre que les neurones dopaminergiques, naturellement colorés en noir du fait de l'auto-oxydation de la dopamine chez les sujets contrôles (panel du haut à gauche) ne le sont plus chez les patients parkinsoniens (panel du haut à droite). La coloration de Pearls, en rose, montre que la $\mathrm{SN}$ est naturellement riche en fer ( $2^{\text {ème }}$ ligne à gauche) mais que cette concentration en fer est augmentée chez les patients ( $2^{\text {ème }}$ ligne à droite). Imagerie par résonance magnétique du mésencéphale. La séquence IRM T2* montre une intensité plus importante $(\mathrm{R} 2 *=1 / \mathrm{T} 2 *)$ chez les patients (en bas à droite) que chez les sujets contrôles (en bas à gauche). L'analyse post-traitement de susceptibilité de distribution quantitative (QSM, 3 Tesla) montre une hyperintensité du signal dans la $\mathrm{SN}$ de patients parkinsoniens (3 ${ }^{\text {ème }}$ ligne à droite) comparé à la SN de sujets sains (3 ${ }^{\text {ème }}$ ligne à gauche). Le noyau rouge peut être identifié au-dessus de la SN. 
FIGURE 2 : La ferroptose comme cible thérapeutique de la maladie de Parkinson. Les altérations des voies métaboliques régulatrices du fer et de l'oxydation phospholipidique sont impliquées dans la maladie de Parkinson. L'augmentation de fer intracellulaire résulte d'une endocytose anormale du fer couplé à la transferrine (Tf) via son récepteur (TfR) et promu par l'alpha-synucléine ( $\alpha$-syn), mais résulte aussi d'une augmentation du taux de $\mathrm{Fe}^{2+}$ via le transporteur divalent de métaux de type 1 (DMT1). Egalement, l'exocytose du fer est dérégulée de part la déstabilisation de la ferroportine (Fpn) à la surface cellulaire par la protéine précurseur $\beta$-amyloide (APP) ou la céruloplasmine (CP). Quand les protéines de stockage du fer que sont la neuromélanine $(\mathrm{Nm})$ et la ferritine $(\mathrm{Ft})$ ne sont plus capables d'assurer son stockage intracellulaire, le pool de fer labile est augmenté, ce qui conduit à la formation de phospholipides hydroperoxydés. L'import de cystéine via le système $X_{c-}$ (dans des conditions d'oxydation) ou le système ASC (Alanine, Sérine, Cystéine) est requis pour la biosynthèse du glutathion (GSH). La glutathione peroxydase de type 4 (Gpx4) utilise 2 molécules de GSH pour réduire les phospholipides peroxydés en leurs composants lipidiques, produisant de l' $\mathrm{H}_{2} \mathrm{O}$ et de la glutathione disulphide (GSSG). Des taux élevés de fer intracellulaire couplés à une déplétion de la Gpx4, comme montré dans les modèles animaux de la MP, provoquent une accumulation de phospholipides hydroperoxydés (LOOH-PL) conduisant à la perte d'intégrité de la membrane cellulaire via une mécanisme ferroptotique. Réduire la quantité de fer labile du pool intracellulaire (grâce à la DFP) ou diminuer la quantité de phospholipides hydroperoxydés (grâce à la liproxstatin-1 ou la ferrostatine-1) sont des cibles prometteuses pour inhiber la ferroptose qui a lieu dans la MP. 
FIGURE 3 : Un modèle de chélation conservatrice du fer basé a- sur la recapture du fer qui s'est accumulé dans les compartiments intracellulaires (organelle, cellule ou tissu), et bsur la redistribution dans d'autre cellule ou compartiment cellulaire soit par chélation, soit par l'intermédiaire de la transferrine circulante. La présence de l'apo-transferrine dans les fluides circulant assure que le fer recapté est conservé puis redéployé dans les zones déficientes en fer. 